\title{
Short Course High Dose Radiotherapy in the Treatment of Anaplastic Thyroid Carcinoma
}

\author{
Mark J. Stavas, ${ }^{1,2}$ Eric T. Shinohara, ${ }^{1}$ Albert Attia, ${ }^{1}$ Matthew S. Ning, \\ Jeffrey M. Friedman, ${ }^{1}$ and Anthony J. Cmelak ${ }^{1}$ \\ ${ }^{1}$ Department of Radiation Oncology, Vanderbilt University Medical Center, Nashville, TN 37232, USA \\ ${ }^{2}$ Department of Radiation Oncology, Vanderbilt University School of Medicine, 1301 Medical Center Drive, B913/TVC, \\ Nashville, TN 37232, USA \\ Correspondence should be addressed to Mark J. Stavas; mark.j.stavas@vanderbilt.edu
}

Received 7 July 2014; Revised 9 September 2014; Accepted 14 September 2014; Published 14 October 2014

Academic Editor: B. C. Stack Jr.

Copyright (C) 2014 Mark J. Stavas et al. This is an open access article distributed under the Creative Commons Attribution License, which permits unrestricted use, distribution, and reproduction in any medium, provided the original work is properly cited.

\begin{abstract}
Purpose. Anaplastic thyroid carcinoma (ATC) is a rare but aggressive tumor with limited survival. To date, the ideal radiation treatment schedule, one that balances limited survival with treatment efficacy, remains undefined. In this retrospective series we investigate the effectiveness and tolerability of hypofractionated radiation therapy in the treatment of ATC. Methods. 17 patients with biopsy proven ATC treated between 2004 and 2012 were reviewed for outcomes and toxicity. All patients received short course radiation. Results. The most commonly prescribed dose was $54 \mathrm{~Gy}$ in 18 fractions. Median survival was 9.3 months. $47 \%$ of patients were metastatic at diagnosis and the majority of patients $(88 \%)$ went on to develop metastasis. Death from local progression was seen in 3 patients (18\%), 41\% experienced grade 3 toxicity, and there were no grade 4 toxicities. Conclusions. Here we demonstrated the safety and feasibility of hypofractionated radiotherapy in the treatment of ATC. This approach offers shorter treatment courses (3-4 weeks) compared to traditional fractionation schedules (6-7 weeks), comparable toxicity, local control, and the ability to transition to palliative care sooner. Local control was dependent on the degree of surgical debulking, even in the metastatic setting.
\end{abstract}

\section{Introduction}

Anaplastic thyroid carcinoma (ATC) is a rare but deadly tumor with a median survival of 5-6 months and less than $20 \%$ survival at one year $[1,2]$. ATC occurs with a slight female predominance $(1.5: 1$ ratio) with a peak incidence in the sixth and seventh decades of life [3]. The majority of patients $(>75 \%)$ develop distant metastasis either at the time of diagnosis or shortly thereafter, with the lungs being the most commonly affected organ [4]. Despite its low incidence, ATC accounts for up to 39\% of thyroid cancer deaths [2] with disease-specific mortality approaching $100 \%$ [5].

In addition to its poor prognosis, ATC causes significant site-specific morbidity. Commonly, individuals present with a rapidly enlarging neck mass causing symptoms of dysphagia, odynophagia, dyspnea, anxiety, and vocal cord paralysis. Unless aggressive treatments are applied, such as surgical resection and external beam radiation therapy (EBRT), patients die from uncontrolled local progression causing suffocation and massive bleeding [6,7]. Furthermore, rapid airway obstruction remains a major cause of death even in patients undergoing tracheostomy $[8,9]$. Therefore, treatment approaches should emphasize the importance of local control even in the metastatic setting.

Given the morbidity and poor prognosis of ATC, palliative and supportive care remain an essential part in the management of these patients $[5,10]$. Owing to disappointing results with current treatments strategies, there have been many attempts to improve clinical outcomes. The most promising one, in terms of local control, has been with trimodality therapy combining surgery with chemotherapy and radiation [11]. Various altered fractionation schedules have been proposed such as twice daily treatments or protracted once daily courses, but these schedules do not consider 
the challenges posed upon patients with limited survival. Two of the main concerns with twice daily treatments are the burden of transportation and association with significant acute toxicities. Furthermore, even with the use of standard fraction sizes and traditional 30-day treatment courses, treatment related side effects tend to be serious, requiring hospitalizations, parenteral nutrition, and delays in supportive care [12].

To date, an optimal radiation approach to ATC (one that considers both outcomes and quality of life) has not been defined. Because of the significant toxicities and logistical challenges related to prolonged or twice daily treatment schedules, our institution has shifted to treat ATC with maximum surgical debulking followed by short course high dose (hypofractionated) radiotherapy with or without chemotherapy. From our experience, shorter treatment courses minimize the challenges associated with transportation and ontreatment toxicity. In addition, shorter courses may lessen the burden of care in this population, especially when the concern for long-term radiation effects is less critical. Most importantly, the quality of life in patients with limited survival should be of highest priority. Here we retrospectively review our use of hypofractionated radiotherapy in the treatment of ATC.

\section{Methods}

2.1. Patients. Following institutional review board approval, patients with pathologically proven ATC referred to the department of radiation oncology between 2004 and 2012 were retrospectively reviewed for outcomes and treatment toxicity. All patients received $\geq 2.5 \mathrm{~Gy}$ dose per fraction (median dose $3 \mathrm{~Gy}$ (range 2.5-4 Gy)). Patients with metastases at the time of diagnosis were included because local control of the primary site was a major issue in this population.

2.2. Treatment. All patients underwent a computed tomography simulation for treatment planning. They were placed in a long thermoplastic mask for immobilization with their neck in maximum extension. The treatment volumes were constructed using presurgical cranial/caudal margins and the postsurgical axial margins including the entire thyroid bed. Typical fields for the primary tumor extended from just below the hyoid bone to the level VI cervical and upper mediastinal lymph nodes (Figure 1). Only the involved and at risk nodal levels were treated. Megavoltage external beam radiation was delivered using intensity modulated radiation therapy (IMRT) and Eclipse planning software. Avoidance structures such as the trachea, esophagus, and spinal cord were contoured and well-defined dose constraints were applied. The maximum spinal cord dose was $\leq 36 \mathrm{~Gy}$ in $3 \mathrm{~Gy}$ fractions. Patients were treated once daily Monday through Friday. Treatment toxicity and follow-up images were reviewed in the medical record.

2.3. Follow-Up and Endpoints. Patients were assessed during treatment and subsequently every $2-4$ weeks after treatment
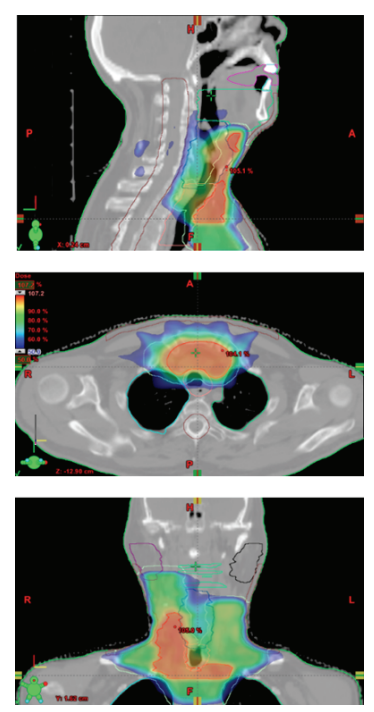

FIGURE 1: Radiation dose distribution for a 65-year-old male with ATC, status post gross total resection with involved cervical nodes. 51 Gy delivered in 17 fractions.

for both response and toxicity. Local control and treatment response were retrospectively evaluated for this analysis based on physical exam and radiographic assessment. Clinically, local control was defined as the absence of physical progression of disease or radiological progression. Physical progression was defined as disease progression requiring any intervention after radiation to protect the airway such as tracheostomy, surgical debulking, or death from upper airway compression. Radiographically, local control was defined as no increase in size greater than $25 \%$ posttreatment. At the time of this analysis, two patients had died during radiotherapy with not enough time to assess treatment response. Both of these patients had unresectable disease and received two fractions of radiation before death. These patients were not included in the outcomes measures. Toxicity was measured according to the National Cancer Institute Common Toxicity Criteria for Adverse Events version 4.0. Toxicity was scored weekly using CTC criteria during the radiation on treatment visit. After completion of treatment, patients were followed up approximately every 4 weeks at Vanderbilt University Medical Center.

2.4. Statistics. All patients who survived treatment were considered eligible for assessment. Outcomes and survival were assessed for all patients. Continuous features were described using means, medians, and ranges whereas categorical features were summarized with frequency counts and percentages. All data were calculated from the time of pathological diagnosis. All patients were followed up until death or last documented follow-up.

\section{Results}

3.1. Patient Characteristics. Seventeen patients were diagnosed with anaplastic thyroid carcinoma and completed 
TABLe 1: Patient demographics.

\begin{tabular}{|c|c|c|c|c|c|c|c|}
\hline Patient & Gender & Age & Date of diagnosis & KPS & Size $(\mathrm{cm})$ & PEG post-XRT & First site of metastasis \\
\hline 1 & $\mathrm{~F}$ & 70 & $1 / 16 / 04$ & 80 & 2.6 & No & Pulmonary \\
\hline 2 & M & 58 & $6 / 30 / 05$ & 80 & 3.2 & No & Pulmonary \\
\hline 3 & $\mathrm{~F}$ & 84 & $4 / 6 / 06$ & 60 & 5.7 & No & Pulmonary \\
\hline 4 & $\mathrm{~F}$ & 60 & $4 / 25 / 06$ & 70 & 4.0 & Yes & Pulmonary \\
\hline 5 & $\mathrm{~F}$ & 77 & $5 / 18 / 06$ & 50 & 5.2 & No & None \\
\hline 6 & $\mathrm{~F}$ & 72 & $9 / 8 / 06$ & 60 & 5.0 & No & None \\
\hline 7 & $\mathrm{~F}$ & 68 & $1 / 2 / 08$ & 80 & 3 & No & Pulmonary \\
\hline 8 & M & 78 & $12 / 19 / 07$ & 70 & 5.9 & Yes & Skin and chest \\
\hline 9 & $\mathrm{~F}$ & 68 & $12 / 7 / 07$ & 80 & 4 & Yes & Pulmonary \\
\hline 10 & M & 77 & $12 / 15 / 08$ & 70 & 5.3 & No & Pulmonary \\
\hline 11 & M & 63 & $4 / 21 / 10$ & 70 & 16 & No & Pulmonary \\
\hline 12 & M & 80 & $9 / 15 / 10$ & 70 & 6.0 & No & Pulmonary \\
\hline 13 & $\mathrm{~F}$ & 76 & $9 / 15 / 10$ & 60 & 6.0 & No & Pulmonary \\
\hline 14 & $\mathrm{M}$ & 62 & $7 / 21 / 11$ & 80 & 2.5 & No & Pulmonary \\
\hline 15 & M & 74 & $8 / 31 / 11$ & 70 & 8.0 & No & Pulmonary \\
\hline 16 & M & 70 & $6 / 11 / 12$ & 70 & 2.5 & Yes & Pulmonary \\
\hline 17 & $\mathrm{~F}$ & 66 & $6 / 21 / 11$ & 80 & 2.2 & No & Pulmonary \\
\hline
\end{tabular}

TABLE 2: Tumor characteristics.

\begin{tabular}{|c|c|c|c|c|c|}
\hline Patient & TNM stage & Margin & Surgery & Nodal dissection & Survival (months) \\
\hline 1 & T4aN0M1 & Negative & Total thyroidectomy & Yes & 21.9 \\
\hline 2 & T4aN0M0 & Positive & Total thyroidectomy & Yes & 23.6 \\
\hline 3 & T4aN0M0 & N/A & None & No & 14.2 \\
\hline 4 & T4aN1aM1 & Gross residual disease & Partial thyroidectomy & No & 4.7 \\
\hline 5 & T4aN1bM0 & Gross residual disease & Partial thyroidectomy & Yes & 2.3 \\
\hline 6 & T4bN0M0 & Negative & Total thyroidectomy & Yes & 24.0 \\
\hline 7 & T4aNlbM1 & Gross residual disease & Partial thyroidectomy & No & 9.3 \\
\hline 8 & T4bN1bM1 & Positive & Total thyroidectomy & Yes & 5.8 \\
\hline 9 & T4aN1bM0 & Positive & Total thyroidectomy & Yes & 10.0 \\
\hline 10 & T4aN1bM1 & Positive & Total thyroidectomy & Yes & 6.5 \\
\hline 11 & T4aN1bM0 & Positive & Partial thyroidectomy & Yes & 23.3 \\
\hline 12 & T4aN1aM1 & $\mathrm{N} / \mathrm{A}$ & None & No & 6.2 \\
\hline 13 & T4aN0M0 & Negative & Total thyroidectomy & No & $17.8^{*}$ \\
\hline 14 & T4aN1bM0 & Positive & Total thyroidectomy & Yes & 4.4 \\
\hline 15 & T4bN0M0 & Positive & Total thyroidectomy & Yes & 7.9 \\
\hline 16 & T4aN1aM1 & $\mathrm{N} / \mathrm{A}$ & None & No & $3.8^{*}$ \\
\hline 17 & T4aN0M1 & Negative & Total thyroidectomy & Yes & $16.1^{*}$ \\
\hline
\end{tabular}

${ }^{*}$ Date of last follow-up.

treatment with hypofractionated radiotherapy between 2004 and 2012. Nine patients were female and 8 were male $(\mathrm{M}: \mathrm{F}$ ratio, 1:1.1). The median age of diagnosis was 70 years (range, 58-84 years). The median Karnofsky performance status was 70 (range, 50-80). Patient and tumor characteristics are described in Tables 1, 2, and 3. All 17 patients had ATC confirmed by pathological review at our institution. The majority of patients (88\%) received concurrent chemotherapy, which consisted of weekly paclitaxel $\left(135 \mathrm{mg} / \mathrm{m}^{2}\right)$ or weekly carboplatin (AUC 1-2) and paclitaxel (135 mg/m $\mathrm{m}^{2}$ ).
3.2. Treatment. Fourteen patients underwent surgical resection prior to radiation $(82.3 \%)$ : ten patients underwent a total thyroidectomy and 4 patients underwent a partial thyroidectomy. Three patients were deemed inoperable at the time of diagnosis: 1 patient due to medical comorbidities and 2 patients due to unresectable disease. The most frequently used fraction size was $3.0 \mathrm{~Gy}$, and the most frequently prescribed dose was 54 Gy in 18 daily fractions (35.3\%) with a range of 40-62.5 Gy (Table 3). The median number of fractions was 18 (range, 10-25). 
TABLE 3: Treatment characteristics.

\begin{tabular}{|c|c|c|c|c|c|c|}
\hline Patient & Elapsed days & Completed treatment & Dose & Fraction & Concurrent chemotherapy & Local control \\
\hline 1 & 29 & Yes with delay & 5100 & 17 & Yes & Yes \\
\hline 2 & 23 & Yes & 5100 & 17 & Yes & Yes \\
\hline 3 & 37 & Yes & 6250 & 25 & No & No \\
\hline 4 & 36 & Yes with delay & 5700 & 19 & Yes & No \\
\hline 5 & 27 & Yes with delay & 5700 & 19 & Yes & Yes \\
\hline 6 & 29 & Yes & 5500 & 22 & Yes & Yes \\
\hline 7 & 23 & Yes & 5400 & 18 & Yes & Yes \\
\hline 8 & 24 & Yes & 5400 & 18 & Yes & Yes \\
\hline 9 & 24 & Yes & 5400 & 18 & Yes & Yes \\
\hline 10 & 13 & Yes & 4000 & 10 & No & Yes \\
\hline 11 & 23 & Yes & 4950 & 18 & Yes & Yes \\
\hline 12 & 23 & Yes & 5400 & 18 & Yes & Yes \\
\hline 13 & 36 & Yes & 6250 & 25 & Yes & Yes \\
\hline 14 & 26 & Yes & 5400 & 18 & Yes & Yes \\
\hline 15 & 22 & Yes & 5100 & 17 & Yes & Yes \\
\hline 16 & 25 & Yes & 5400 & 18 & Yes & No \\
\hline 17 & 21 & Yes & 4500 & 15 & Yes & Yes \\
\hline
\end{tabular}

TABLE 4: Toxicity.

\begin{tabular}{lcccc}
\hline Grade & & Toxicity/number of patients (\%) & Dermatitis & Total $(N)$ \\
\hline 1 & Dysphagia & Esophagitis & $7(45)$ & $17(100)$ \\
2 & $7(45)$ & $9(53)$ & $6(35)$ & $17(100)$ \\
3 & $6(35)$ & $5(29)$ & $4(24)$ & $7(41)$ \\
4 & $4(24)$ & $3(18)$ & 0 & 0 \\
\hline
\end{tabular}

3.3. Local Control and Survival. The median survival was 9.3 months. For those with metastatic disease at diagnosis, the median survival was 6.4 months; for those without initial metastases, median survival was 14.2 months. Seven of the 17 patients $(41 \%)$ were alive one year after initial diagnosis. Two of the 8 patients with metastatic disease at diagnosis were alive after one year, while 5 of the 9 patients (56\%) without initial metastases were alive after one year.

Eight patients (47\%) had metastatic disease at the time of diagnosis. The median time from diagnosis to metastasis was 2.1 months, and $88 \%$ of irradiated patients developed metastatic disease. The lungs were the first site of metastasis in $93 \%$ of patients. Fourteen patients had died at the time of analysis. Three patients were lost to follow-up after a mean of 12.5 months. These patients were assumed dead with survival calculated from the last date of follow-up.

Fourteen of the 17 patients ( $82 \%$ ) maintained local control of disease at the time of death. Two patients required palliative tracheostomies and one patient required surgical debulking after irradiation. In the patients who experienced local progression, 2 had unresectable disease at diagnosis and 1 received a partial thyroidectomy. Four patients required percutaneous gastrostomy tube placement following radiation. Radiation induced esophagitis was the reason for gastrostomy tube placement in 2 of 4 patients.
3.4. Toxicity and Feasibility. Treatment related toxicities were graded using the National Cancer Institute Common Toxicity Criteria for Adverse Events version 4.0. Specifically, dysphagia, esophagitis, and radiation dermatitis were analyzed as documented in the medical record. The incidences of grades 1-4 toxicities are listed in Table 4 . All patients experienced grade 1 or 2 toxicities. There were no grade 4 toxicities. However, 7 out of 17 patients (41\%) experienced at least one grade 3 toxicity. Moreover, patients with grade 3 toxicity in one category were more likely to have grade 3 toxicity in another category $(\mathrm{HR}=1.33)$. Frequently, this was the result of symptomatic overlap between esophagitis and dysphagia. Patients who were deemed unresectable at diagnosis or underwent a partial resection prior to radiation were more likely to develop grade 3 dysphagia during treatment. This appeared to be related to local tumor progression resulting in mechanical obstruction as opposed to radiation induced esophagitis. Patients who underwent a complete resection prior to the start of radiation experienced the fewest toxicities and treatment delays.

All patients completed their radiation course as prescribed. A treatment delay was defined as an interruption between two radiation fractions greater than 5 days or a total treatment time 10 days greater than expected. Three out of 17 patients experienced treatment delays (18\%). All 
three patients required inpatient admission with a mean hospitalization time of 3.6 days. Reasons for a treatment delay were local progression requiring tracheostomy or poor nutritional intake requiring gastrostomy tube placement.

\section{Discussion}

Given the poor prognosis and rapid progression of this disease, early integration of palliative and supportive care is essential when managing these patients. Knowing when and what type of radiation treatment to deliver can be a clinical challenge and requires a comprehensive understanding of the disease's natural history, sequelae of symptoms, treatment effectiveness, and goals of care. Unfortunately, patients with ATC are confronted with an aggressive disease that affects critical respiratory organs and local control remains a primary determinant of quality of life even in the metastatic setting. Results from the present study appear similar to prior studies with regard to local control and survival (Table 5).

Similar to other reports, our data underline the importance of surgical debulking in the management of ATC. Junor et al. reported that patients who underwent a total or partial thyroidectomy had prolonged survival times compared with patients for whom only a biopsy was feasible [7]. Surgical debulking can prevent distressing symptoms such as airway compression and it is recommended even in the presence of metastasis [16, 17]. Furthermore, reducing the burden of local disease may improve the efficacy of adjuvant therapy. However, surgery alone cannot alter the course of this disease [18]. The combination of surgery and radiotherapy is an independent predictor of reduced cause-specific mortality in patients with ATC [19], though the ideal adjuvant radiation regimen remains unclear.

The low incidence and poor survival rates of patients with ATC limit the ability to conduct large Phase III trials. Most of the available evidence for radiotherapy is derived from single institutional retrospective series. Wang et al. reported on 47 patients with ATC who received radiotherapy as either once or twice daily fractionation escalating up to $66 \mathrm{~Gy}$. Median survival was 5.6 months, but patients receiving higher doses of radiation (45-66 Gy) had significantly longer survival times compared to those receiving doses less than $40 \mathrm{~Gy}$ (11.1 versus 3.2 months; $P<0.001$ ) [13]. In our series, delivering larger doses in a shorter period of time maintained the concept of dose escalation, yielding an average biologically equivalent dose of $70.2 \mathrm{~Gy}$.

Several common treatment schedules are used in radiation oncology including once daily treatments for $\sim 25-35$ days, twice daily treatments for $\sim 15-25$ days, and hypofractionated treatments for $\sim 1-20$ days. Typically, radiation oncologists attempt to achieve the same total effective dose; therefore, if the number of treatments decreases, the total dose per treatment must increase or the dose is delivered twice daily. One of the major challenges with twice daily treatments is the 6-hour break between fractions. The patient spends most of the day in the clinic or arranging transportation to and from it. The second challenge is increased acute toxicity, which is an important consideration when treating the head and neck region.

Toxicity is divided into acute and late-responding effects depending on whether the tissue is more likely to manifest radiation damage around the time of treatment or in the future. Larger radiation doses per day correspond to greater risk of damage in late-responding tissues (such as the spinal cord) as compared to smaller doses given over a protracted course. However, in patients with limited survival, shorter treatment courses are practical because the patient will not live long enough to face the increased risk of long-term side effects, which classically occur many months to years later.

In the pursuit of better outcomes, several studies have examined the use of twice daily accelerated radiotherapy. De Crevoisier demonstrated treatment effectiveness, but 33\% of their patients experienced grade III or IV acute mucositis with a significant amount of chemotherapy induced hematologic toxicity [14]. Dandekar et al. reported that greater than $70 \%$ of their patients experienced grade III or IV acute dysphagia and esophagitis and many of these patients discontinued treatment, with less than $10 \%$ survival at one year [20].

In our series, toxicity with hypofractionated radiotherapy continued to be an issue with $41 \%$ of patients experiencing grade 3 toxicities, but no grade 4 toxicities. The important difference between this regimen and others was that acute toxicities were seen near the end of treatment or after the patient had finished their radiation, thereby limiting the number of treatment related breaks and total treatment time. This allowed patients to continue forward with adjuvant systemic therapy or palliative and supportive care as needed. From the perspective of patient comfort, shorter treatment courses are preferable to longer courses, especially in the setting of limited survival.

Local recurrence in this disease can have devastating consequences and patients have a median survival of 66 days after local progression (McIver et al. anaplastic thyroid carcinoma: a 50-year experience at a single institution, 2001). This study also demonstrated that radiation after complete or near complete resection did not improve local control but did delay the time to local progression (5 versus 3 months). However, radiation or surgery did appear to improve survival over palliative care alone. In previous studies by Tennvall et al. and Werner et al., death from local failure was seen in $36 \%$ and $24 \%$ of patients, respectively. These studies used twice daily treatment schedules of varying doses and chemotherapy regimens [11, 21]. In our study, death attributed to local failure was seen in $18 \%$ of patients, though the patients who developed local failure were inoperable at the time of diagnosis or only received a partial resection or biopsy. Similarly, Foote et al. achieved local control in 30\% of patients with variable radiation schedules and dual chemotherapy; however, patients with metastatic disease at diagnosis were excluded from their study [22]. These patients account for a significant portion of patients with ATC and were included in our series. Results from the present study suggest that a hypofractionated regimen is as effective as hyperfractionated regimens and while treatment courses were slightly longer, patients were only treated once per day. 


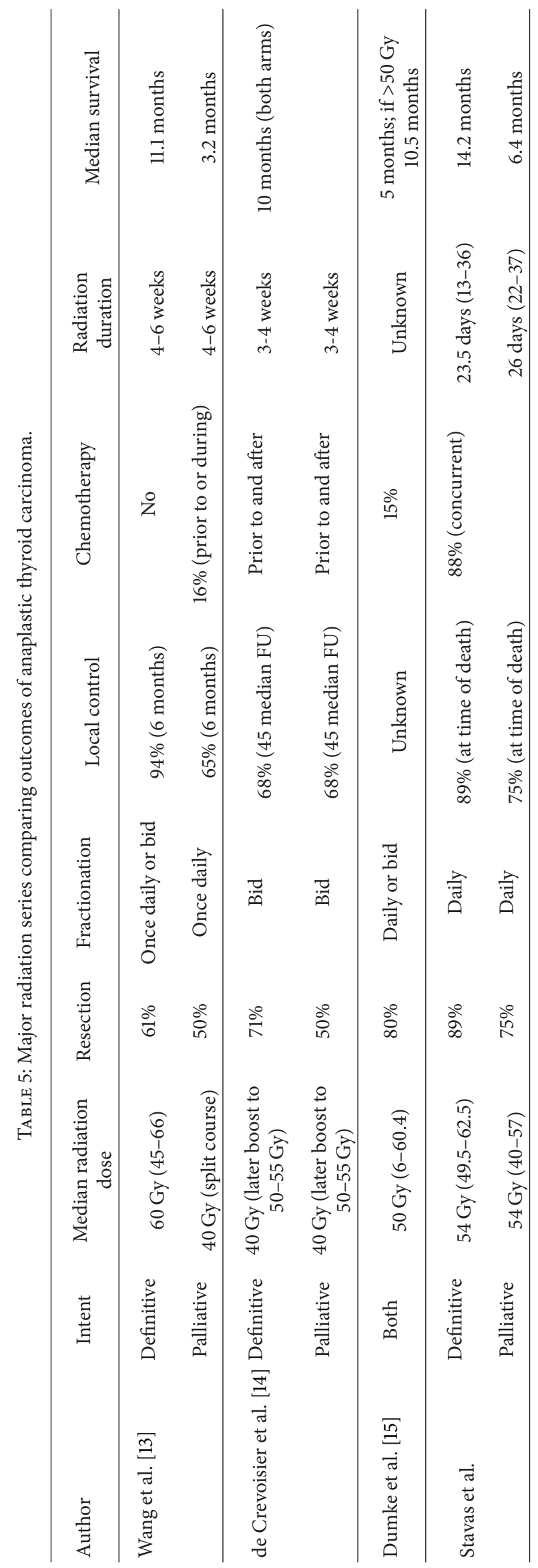


There were multiple limitations in this study including the single institutional retrospective nature, small patient numbers, and variable radiation doses. Most patients received upfront resection possibly because of smaller tumors. Therefore, selection bias may have influenced the overall survival. Despite these issues, this treatment approach is unreported in the literature. Results from the present study suggest that a hypofractionated regimen with concurrent chemotherapy is well tolerated with a favorable toxicity profile and rates of local control when compared with previously used fractionation schemes. While there was no formal assessment of quality of life, given the favorable toxicity as well as the convenience of a shorter, once a day treatment regimen, we believe that this regimen may improve the quality of life in these patients with a generally poor outcome. Based on our results, short course hypofractionated radiation therapy appears to be a viable and safe option in the treatment of ATC and remains a sensible approach given the poor prognosis and symptomatic needs of this patient population. Specifically, this protocol may be useful in patients who have difficulty with a twice daily regimen due to either travel or concerns about their ability to tolerate the acute effects of a twice daily regimen.

\section{Conflict of Interests}

The authors declare that there is no conflict of interests regarding the publication of this paper.

\section{References}

[1] F. D. Gilliland, W. C. Hunt, D. M. Morris, and C. R. Key, "Prognostic factors for thyroid carcinoma. A population-based study of 15, 698 cases from the Surveillance, Epidemiology and End Results (SEER) program 1973-1991," Cancer, vol. 79, pp. 564$573,1997$.

[2] S. A. Hundahl, I. D. Fleming, A. M. Fremgen, and H. R. Menck, "A National Cancer Data Base report on 53, 856 cases of thyroid carcinoma treated in the U.S., 1985-1995," Cancer, vol. 83, pp. 2638-2648, 1998.

[3] K. B. Ain, "Anaplastic thyroid carcinoma: behavior, biology, and therapeutic approaches," Thyroid, vol. 8, no. 8, pp. 715-726, 1998.

[4] C. Are and A. R. Shaha, "Anaplastic thyroid carcinoma: biology, pathogenesis, prognostic factors, and treatment approaches," Annals of Surgical Oncology, vol. 13, no. 4, pp. 453-464, 2006.

[5] R. L. Neff, W. B. Farrar, R. T. Kloos, and K. D. Burman, "Anaplastic thyroid cancer," Endocrinology and Metabolism Clinics of North America, vol. 37, no. 2, pp. 525-538, 2008.

[6] B. Jereb, J. Stjernsward, and T. Lowhagen, "Anaplastic giant cell carcinoma of the thyroid. A study of treatment and prognosis," Cancer, vol. 35, no. 5, pp. 1293-1295, 1975.

[7] E. J. Junor, J. Paul, and N. S. Reed, "Anaplastic thyroid carcinoma: 91 patients treated by surgery and radiotherapy," European Journal of Surgical Oncology, vol. 18, no. 2, pp. 83-88, 1992.

[8] R. K. Tan, R. K. Finley III, D. Driscoll, V. Bakamjian, W. L. Hicks Jr., and D. P. Shedd, "Anaplastic carcinoma of the thyroid: a 24year experience," Head and Neck, vol. 17, no. 1, pp. 41-48, 1995.
[9] E. Tallroth, G. Wallin, G. Lundell, T. Löwhagen, and J. Einhorn, "Multimodality treatment in anaplastic giant cell thyroid carcinoma," Cancer, vol. 60, no. 7, pp. 1428-1431, 1987.

[10] R. C. Smallridge, K. B. Ain, S. L. Asa et al., "American Thyroid Association guidelines for management of patients with anaplastic thyroid cancer," Thyroid, vol. 22, pp. 1104-1139, 2012.

[11] J. Tennvall, G. Lundell, P. Wahlberg et al., "Anaplastic thyroid carcinoma: three protocols combining doxorubicin, hyperfractionated radiotherapy and surgery," British Journal of Cancer, vol. 86, no. 12, pp. 1848-1853, 2002.

[12] M. Troch, O. Koperek, C. Scheuba et al., "High efficacy of concomitant treatment of undifferentiated (anaplastic) thyroid cancer with radiation and docetaxel," The Journal of Clinical Endocrinology and Metabolism, vol. 95, no. 9, pp. E54-E57, 2010.

[13] Y. Wang, R. Tsang, S. Asa, B. Dickson, T. Arenovich, and J. Brierley, "Clinical outcome of anaplastic thyroid carcinoma treated with radiotherapy of once- and twice-daily fractionation regimens," Cancer, vol. 107, no. 8, pp. 1786-1792, 2006.

[14] R. de Crevoisier, E. Baudin, A. Bachelot et al., "Combined treatment of anaplastic thyroid carcinoma with surgery, chemotherapy, and hyperfractionated accelerated external radiotherapy," International Journal of Radiation Oncology Biology Physics, vol. 60, no. 4, pp. 1137-1143, 2004.

[15] A.-K. Dumke, T. Pelz, and D. Vordermark, "Long-term results of radiotherapy in anaplastic thyroid cancer," Radiation Oncology, vol. 9, no. 1, article 90, 2014.

[16] P. I. Haigh, P. H. Ituarte, H. S. Wu et al., "Completely resected anaplastic thyroid carcinoma combined with adjuvant chemotherapy and irradiation is associated with prolonged survival," Cancer, vol. 91, pp. 2335-2342, 2001.

[17] O. Nilsson, J. Lindeberg, J. Zedenius et al., "Anaplastic giant cell carcinoma of the thyroid gland: treatment and survival over a 25-year period," World Journal of Surgery, vol. 22, no. 7, pp. 725730, 1998.

[18] B. McIver, I. D. Hay, D. F. Giuffrida et al., "Anaplastic thyroid carcinoma: a 50-year experience at a single institution," Surgery, vol. 130, no. 6, pp. 1028-1034, 2001.

[19] E. Kebebew, F. S. Greenspan, O. H. Clark, K. A. Woeber, and A. McMillan, "Anaplastic thyroid carcinoma: treatment outcome and prognostic factors," Cancer, vol. 103, no. 7, pp. 1330-1335, 2005.

[20] P. Dandekar, C. Harmer, Y. Barbachano et al., "Hyperfractionated Accelerated Radiotherapy (HART) for anaplastic thyroid carcinoma: toxicity and survival analysis," International Journal of Radiation Oncology Biology Physics, vol. 74, no. 2, pp. 518-521, 2009.

[21] B. Werner, J. Abele, A. Alveryd et al., "Multimodal therapy in anaplastic giant cell thyroid carcinoma," World Journal of Surgery, vol. 8, no. 1, pp. 64-68, 1984.

[22] R. L. Foote, J. R. Molina, J. L. Kasperbauer et al., "Enhanced survival in locoregionally confined anaplastic thyroid carcinoma: a single-institution experience using aggressive multimodal therapy," Thyroid, vol. 21, no. 1, pp. 25-30, 2011. 


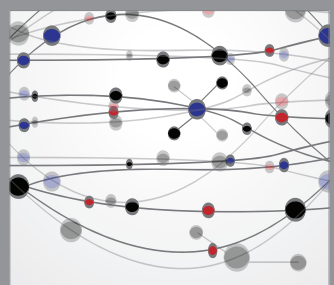

The Scientific World Journal
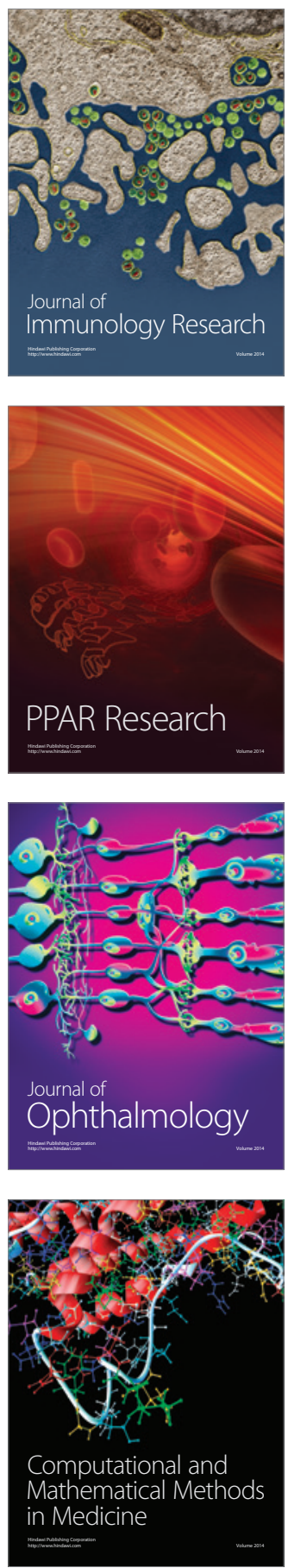

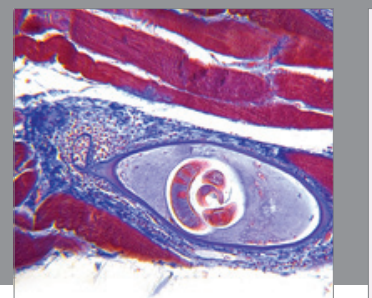

Gastroenterology

Research and Practice
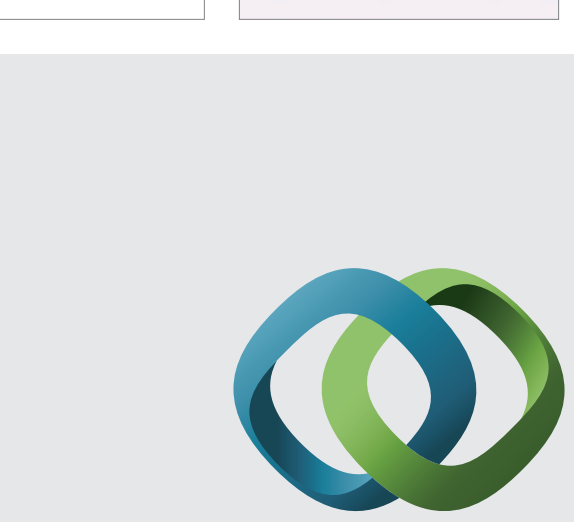

\section{Hindawi}

Submit your manuscripts at

http://www.hindawi.com
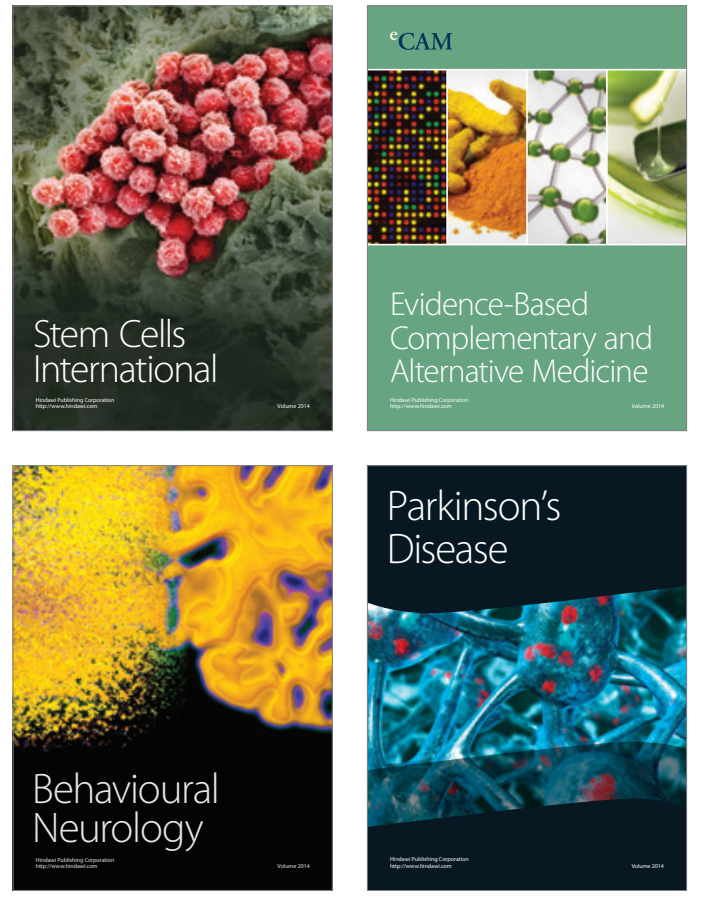
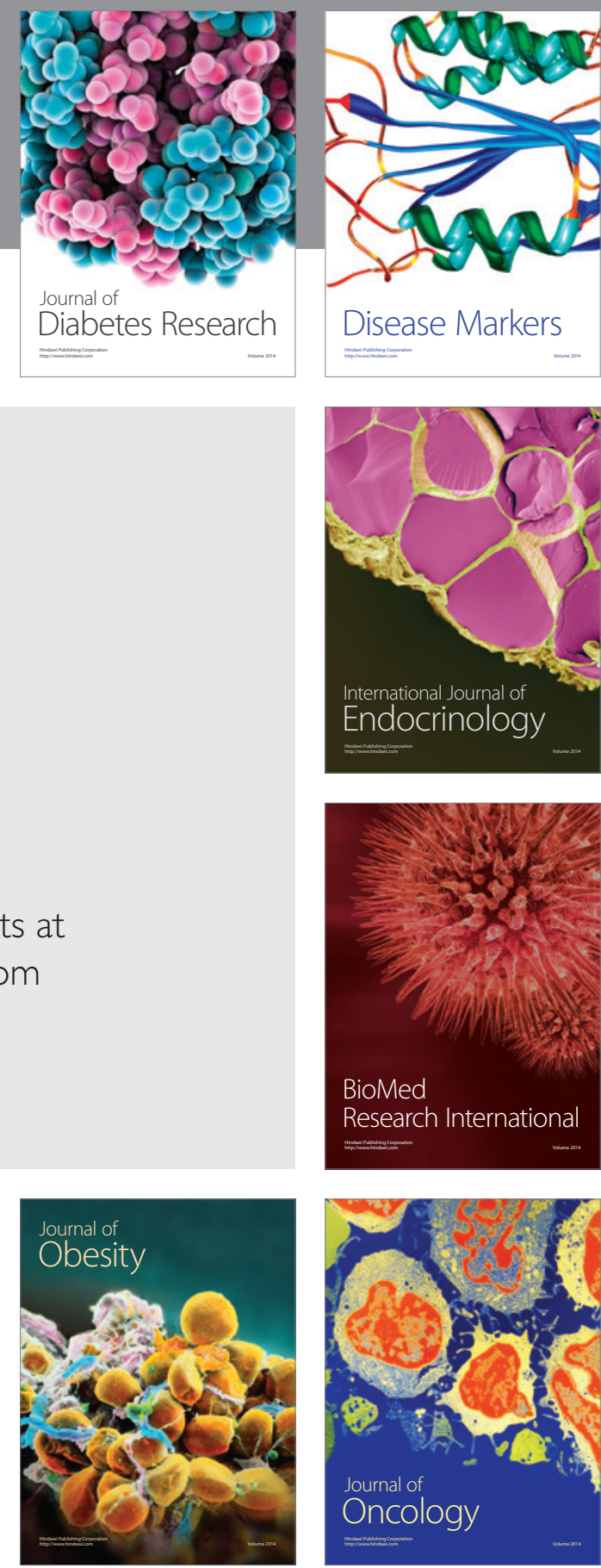

Disease Markers
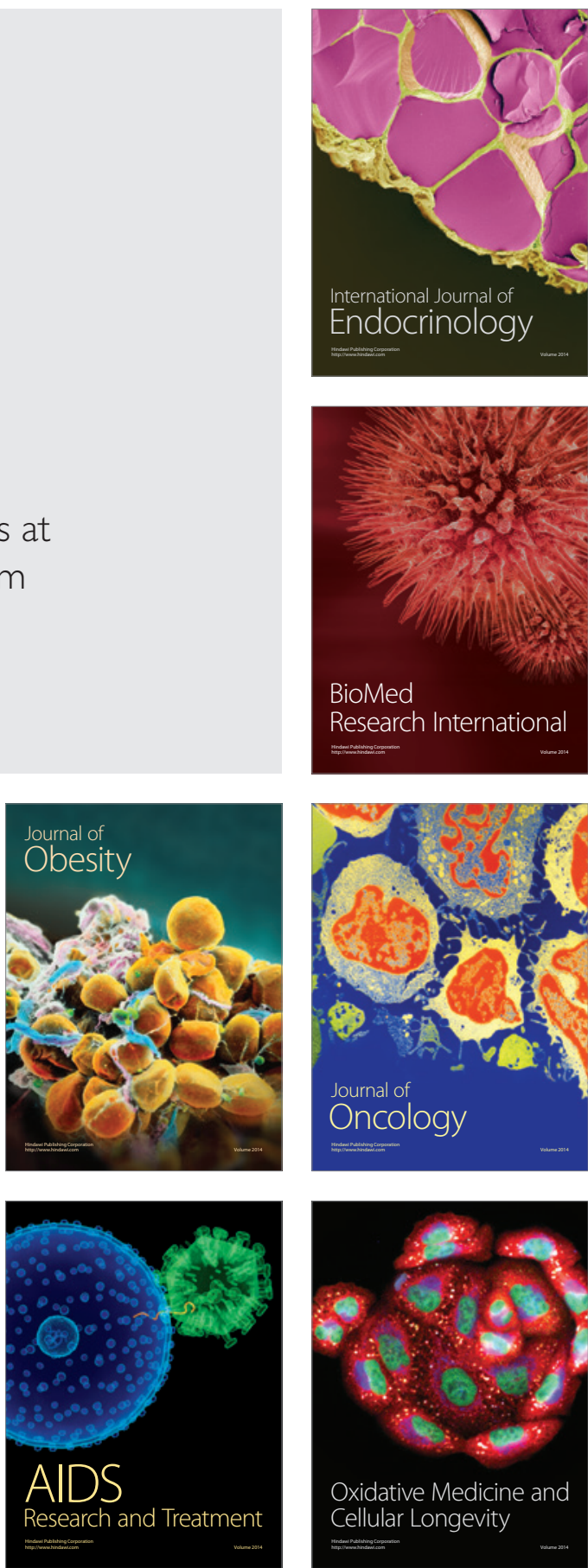